\title{
Arrasto hidrodinâmico activo e potência mecânica máxima em nadadores pré-juniores de Portugal
}

\author{
J. P. Vilas-Boas \\ R. Fernandes \\ Faculdade de Ciências do Desporto e de Educação Física \\ Universidade do Porto \\ Portugal
}

https://doi.org/10.5628/rpcd.01.03.14

\section{RESUMO}

O objectivo do presente estudo consistiu em caracterizar o arrasto hidrodinâmico activo máximo (D) e a potência mecânica máxima $(\mathrm{P})$ na técnica de crawl em jovens nadadores portugueses de ambos os sexos e de elevado potencial desportivo. Como objectivo secundário procurou-se comparar os respectivos valores com os de nadadores de elevado nível nacional e com outros de elite internacional. Foram estudados 61 nadadores pré-juniores, 29 do sexo masculino (15-16 anos) e 32 do sexo feminino (13-14 anos). Como amostras de referência utilizaram-se $n=5$ nadadores e $n=3$ nadadoras juniores e seniores nacionais de bom nível desportivo e $\mathrm{n}=3$ nadadores de elite internacional. $\mathrm{D}$ e o coeficiente de arrasto $\left(\mathrm{C}_{\mathrm{D}}\right)$ foram determinados pelo método da potência máxima constante e a potência foi determinada pelo produto da intensidade da força de arrasto pela velocidade de nado. Os resultados permitiram concluir que: (i) os valores de $\mathrm{D}$ à velocidade máxima são inferiores nos nadadores mais jovens e nos nadadores masculinos de top internacional; (ii) os valores de $\mathrm{D}$ à velocidade máxima de nado, bem como desta, são mais elevados nos nadadores do que nas nadadoras; (iii) os valores de $C_{D}$ tendem a ser superio-res nos nadadores pré-juniores relativamente às nadadoras do mesmo escalão e não variam expressivamente com a idade e nível desportivo e (iv) $\mathrm{P}$ cresce entre os nadadores com a idade e nível desportivo e é superior nos nadadores relativamente às nadadoras.

Palavras-chave: Natação, biomecânica, arrasto activo, potência mecânica

\author{
S. Kolmogorov
}

Faculty of Physical Education

Pomor International University

Arkhangelsk

Russia

\author{
ABSTRACT \\ Active Drag and Maximal Mechanical Power in Juvenile \\ Portuguese Swimmers.
}

The aim of this research was to characterize the active drag force $(D)$ and the maximal propulsive power $(P)$ of juvenile Portuguese swimmers of high performance potencial. As a secondary purpose, we compared these characteristics with those of Portuguese top level swimmers, and international elite swimmers. 61 pre-junior Portuguese swimmers were studied, 29 males (15-16 years old) and 32 females (13-14 years old). As reference samples we used 5 males and 3 females of Portuguese top level, and 3 swimmers of international elite. D force and drag coefficient $\left(C_{D}\right)$ were assessed through the method of constant maximal propulsive power. This late was calculated through the product of drag and swimming velocity. Results pointed out that: (i) D values, at maximal swimming velocity, are lower in younger swimmers and in international top level ones; (ii) D values, at maximal swimming velocity, and maximal swimming velocity itself, were higher for males than for females; (iii) $C D$ values tended to be higher for males than for female counterparts, and seem to do not change with age and performance level; (iv) P grows with age, and tend to be higher in male, than in female swimmers.

Keywords: Swimming, biomechanics, active drag, mechanical power. 


\section{INTRODUÇÃO}

Para que um nadador se desloque no meio aquático de forma continuada é necessário que, a cada momento, seja capaz de produzir uma força propulsiva $\mathrm{p}$ de intensidade pelo menos igual à da força de arrasto hidrodinâmico D que a água opõe ao seu deslocamento. No caso de $\mathrm{p}<\mathrm{D}$, a massa do nadador fica sujeita, no intervalo de tempo em que tal aconteça, a um impulso negativo, que induzirá uma aceleração negativa do movimento e que, em última análise, implicará a imobilização do sujeito (Figura 1).

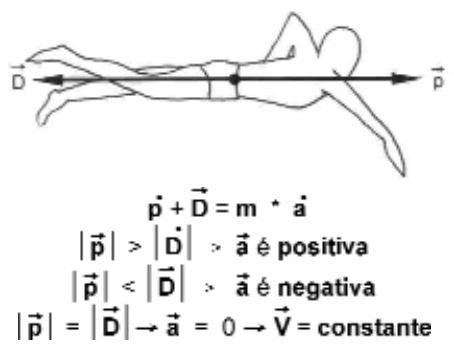

Figura 1. Principais forças em presença e respectivas relações na equação do movimento do nadador. $p=$ força propulsiva; $D=$ arrasto hidrodinâmico; $m$ = massa do nadador + mass $a$ de água acrescentada; $a$ = aceleração; $V=$ velocidade.

Se a capacidade propulsiva consiste numa das competências fundamentais do nadador, na qual se entrecruzam as capacidades técnicas e as qualidades físicas que sustentam a expressão mecânica da força, a capacidade de minimizar D não é menos importante; isto, apesar de se poder considerar que $\mathrm{D}$, relativamente a $\mathrm{p}$, seja menos dependente da técnica e mais determinada por factores constitucionais e, por isso, mais estável.

A importância de D no quadro do complexo de factores biomecânicos, e outros, que constrangem a capacidade de rendimento do nadador é naturalmente reconhecida pela generalidade das comunidades técnica e científica ligadas à natação. De facto, desde há muito que se procura avaliar a intensidade de D em nadadores (12), mas a complexidade da tarefa determinou que se fossem desenvolvendo métodos, ou muito distantes das situações de nado propriamente ditas, ou que implicavam procedimentos complexos e morosos, ao mesmo tempo que envolviam equipamentos delicados e de custo e sofisticação elevados.

Na literatura especializada distinguem-se fundamentalmente duas grandes categorias de métodos para a determinação do arrasto: (i) arrasto passivo $(7,12)$, que se consubstanciam na determinação da força requerida para fazer deslocar o nadador numa determinada posição que é mantida constante e; (ii) arrasto activo $(3,8,5,10,15)$, que procuram avaliar a intensidade de $\mathrm{D}$ a que efectivamente se sujeita o nadador ao nadar livremente, ou tão livremente quanto possível.

Apesar de algumas opiniões em favor da ainda actual utilidade da avaliação do arrasto passivo (1), o interesse da comunidade científica tem recaído predominantemente sobre os métodos de determinação do arrasto activo.

Considerando a relevância de $\mathrm{D}$ e da potência mecânica máxima susceptível de ser desenvolvida pelo trabalho propulsivo do nadador na estrutura de competências que determinam a sua capacidade de rendimento, o objectivo do presente estudo consistiu em caracterizar o arrasto hidrodinâmico activo máximo e a potência mecânica máxima na técnica de crawl em jovens nadadores portugueses de ambos os sexos e de elevado potencial desportivo. Como objectivo secundário procurou-se comparar os respectivos valores com os de nadadores de elevado nível nacional e com outros de elite internacional.

\section{MATERIAL E MÉTODOS}

\subsection{Amostra e grupos de referência}

A amostra foi constituída por um total de 61 sujeitos, 29 do sexo masculino e 32 do sexo feminino (Quadro 1). As idades de todos os nadadores, no momento da avaliação, estavam compreendidas nos escalões etários relativos aos dois anos de categoria nacional Pré-junior (masculinos 15-16 anos e femininos 13-14 anos). 
Quadro 1. Principais características da amostra. Os valores são média \pm desvio-padrão.

\begin{tabular}{|c|c|c|c|c|}
\hline Grupo & Idade [meses] & Peso $[\mathrm{Kg}]$ & Altura $[\mathrm{cm}]$ & Nível desportivo \\
\hline \multicolumn{5}{|c|}{ Masc. $1^{\circ}$ ano } \\
\hline$(n=10)$ & $181.4 \pm 2.72$ & $58.9 \pm 8.69$ & $169.3 \pm 7.38$ & Selecção Pré-junior \\
\hline \multicolumn{5}{|c|}{ Masc. $2^{\circ}$ ano } \\
\hline$(n=19)$ & $193.3 \pm 4.22$ & $61.2 \pm 8.03$ & $171.9 \pm 7.34$ & Selecção Pré-junior \\
\hline \multicolumn{5}{|c|}{ Fem. $1^{\circ}$ ano } \\
\hline$(n=9)$ & $158.6 \pm 1.60$ & $50.9 \pm 5.36$ & $161.8 \pm 3.77$ & Selecção Pré-junior \\
\hline \multicolumn{5}{|c|}{ Fem. $2^{\circ}$ ano } \\
\hline$(n=21)$ & $169.2 \pm 3.71$ & $49.3 \pm 8.63$ & $159.7 \pm 8.34$ & Selecção Pré-junior \\
\hline \multicolumn{5}{|c|}{ Jun. e Sen. Masc. } \\
\hline$(n=5)$ & $235.2 \pm 50.90$ & $75.9 \pm 5.25$ & $183.2 \pm 4.09$ & Top nacional \\
\hline \multicolumn{5}{|c|}{ Jun. e Sen. Fem. } \\
\hline$(n=3)$ & $195.0 \pm 19.80$ & $52.3 \pm 0.04$ & $166.5 \pm 3.54$ & Top nacional \\
\hline \multicolumn{5}{|c|}{ Top Masc. } \\
\hline$(n=3)$ & $294.0 \pm 49.0$ & $80.2 \pm 6.75$ & $190.0 \pm 8.99$ & Top mundial \\
\hline
\end{tabular}

Como amostras de referência utilizaram-se $n=5$ nadadores e $\mathrm{n}=3$ nadadoras juniores e seniores nacionais de bom nível desportivo (top nacional) e $\mathrm{n}=3$ nadadores de elite internacional (top mundial).

\subsection{Situação teste}

Cada nadador realizou dois percursos de $25 \mathrm{~m}$ crawl com partida de dentro de água (situações 1 e 2), em águas estacionárias, numa piscina coberta e aquecida (temperatura da água de $26^{\circ} \mathrm{C}$ ). Foi solicitado a cada nadador que, em cada um dos percursos de $25 \mathrm{~m}$, acelerasse progressivamente até aos $10 \mathrm{~m}$, momento onde deveria estar já animado da sua velocidade máxima, a qual deveria manter até à parede testa de chegada. Entre as duas repetições foi observado um intervalo de, pelo menos, $20 \mathrm{mn}$ de repouso passivo. Em cada percurso foram cronometrados $13 \mathrm{~m}$ de nado puro, sem influência quer do impulso na parede, quer da chegada. Estes $13 \mathrm{~m}$ foram marcados por referências físicas externas, iniciando-se $11 \mathrm{~m}$ após a parede testa de origem e terminando aos $24 \mathrm{~m}$ (Figura 2).

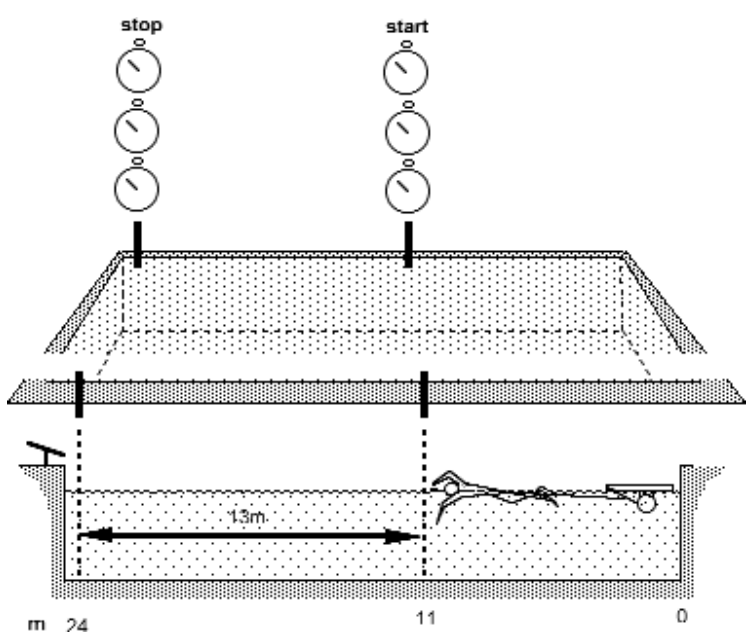

Figura 2. Representação esquemática da situação teste utilizada para a avaliação do arrasto activo. 
As cronometragens foram realizadas através de cronómetros digitais com aproximação às centésimas de segundo, por três cronometristas experientes. Dos três tempos, foi seleccionado o tempo intermédio ou o tempo repetido, não sendo consideradas as repetições onde se observassem discrepâncias superiores a $0.3 \mathrm{~s}$ entre os três cronometristas. O erro associado à cronometragem manual antes descrita foi estimado em $0.8 \%$ (10).

Na primeira repetição (situação 1) cada nadador nadou livremente, enquanto que na segunda (situação 2) rebocou um corpo de propriedades hidrodinâmicas conhecidas (Figura 3).

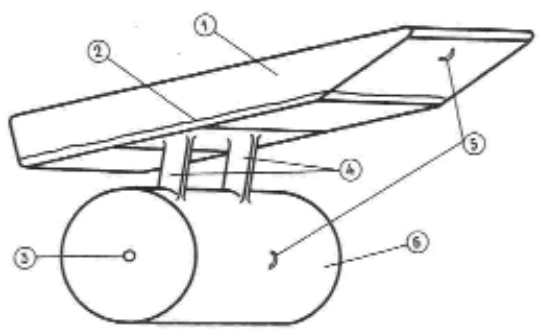

Figura 3. Representação esquemática do corpo hidrodinâmico utilizado na experiência (10). 1 = porção flutuante; 2 = linha da água; 3 =orifício de enchimento; 4 = suportes do cilindro; 5 = locais de ancoragem do cabo; 6 = cilindro hidrodinâmico.

O corpo encontrava-se distanciado $10 \mathrm{~m}$ do nadador, minimizando a influência hidrodinâmica da esteira produzida pelo sujeito, e ligado à cintura deste por um cabo de reduzidas propriedades elásticas. A fixação à cintura do nadador foi conseguida através de um cinto convencional. A distância crítica a que o corpo hidrodinâmico deveria deslocar-se relativamente aos pés do nadador, por forma a não sobrarem interferências na sua hidrodinâmica particular, foi estimada em 3.5 a 4.5 vezes a altura do nadador (10).

\subsection{Método de determinação do arrasto activo}

O método utilizado para a determinação de $\mathrm{D}$ foi anteriormente descrito por Kolmogorov e Duplishcheva (10) e baseia-se no princípio da conservação da potência mecânica propulsiva máxima
(P) do nadador em duas situações de nado distintas, ambas à velocidade máxima: nado livre $\left(\mathrm{P}_{1}\right)$ e nado rebocando um dispositivo de arrasto adicional conhecido $\left(\mathrm{P}_{2}\right)$ :

$$
\mathrm{P}_{1}=\mathrm{P}_{2}
$$

Assumindo a velocidade de nado como constante, $\mathrm{P}_{1}$ pode ser descrita como:

e $\mathrm{P}_{2}$ como:

$$
\mathrm{P}_{1}=\mathrm{D}_{1}^{*} \mathrm{~V}_{1}
$$

$$
\mathrm{P}_{2}=\mathrm{D}_{2} * \mathrm{~V}_{2}
$$

onde $\mathrm{D}_{1}$ corresponde ao arrasto activo na situação 1 e $\mathrm{D}_{2}$ corresponde ao arrasto activo total na situação 2 , ou seja, $\mathrm{D}_{1}$ adicionado ao arrasto conhecido do corpo hidrodinâmico $\left(\mathrm{D}_{\mathrm{ch}}\right)$.

Utilizando as equações newtonianas que descrevem $\mathrm{D}_{1}$ e $\mathrm{D}_{2}$, onde $\mathrm{C}_{\mathrm{D}}$ é o coeficiente de arrasto do corpo, $\rho$ a massa específica da água e $S$ a área de secção máxima do corpo transversal à direcção de $\mathrm{D}$, obtemos:

$$
\mathrm{D}_{1}=\frac{1}{2} \mathrm{C}_{\mathrm{D}}^{*} \rho^{*} \mathrm{~S}^{*} \mathrm{~V} \frac{2}{1}
$$

$\mathrm{e}$

$$
\mathrm{D}_{2}=\frac{1}{2} \mathrm{C}_{\mathrm{D}}^{*} \rho^{*} \mathrm{~S}^{*} \mathrm{~V}_{\frac{2}{2}}+\mathrm{D}_{\mathrm{Ch}}
$$

Combinando as expressões (e1) a (e5), obtém-se uma nova descrição da igualdade (e1):

$$
\frac{1}{2} \mathrm{C}_{\mathrm{D}}^{*} \rho^{*} \mathrm{~S}^{*} \frac{\mathrm{V}}{1}=\frac{1}{2} \mathrm{C}_{\mathrm{D}}^{*} \rho * \mathrm{~S}^{*} \mathrm{~V} \frac{3}{2}+\mathrm{D}_{\mathrm{Ch}}{ }^{*} \mathrm{~V}_{2}
$$

Desenvolvendo (e6) em ordem a CD obtém-se:

$$
C_{\mathrm{D}}=\frac{\mathrm{D}_{\mathrm{Ch}}{ }^{*} \mathrm{~V}_{2}}{\frac{1}{2} \rho^{*} \mathrm{~S}^{*}\left(\mathrm{~V}_{\left.\frac{3}{1}-\mathrm{V}^{\frac{3}{2}}\right)}\right.}
$$

Substituindo CD na expressão (e4) vem:

$$
\mathrm{D}_{1}=\frac{\mathrm{D}_{\mathrm{Ch}}{ }^{*} \mathrm{~V}_{2} * \mathrm{~V}_{1}^{\frac{2}{1}}}{\left.\mathrm{~V} \frac{3}{1}-\mathrm{V}_{3}\right)}
$$


equação que nos proporciona a medida do arrasto activo (D) na situação 1: natação livre à máxima velocidade.

Para além do cálculo de $\mathrm{D}$, o raciocínio desenvolvido antes permite calcular a potência mecânica propulsiva máxima - expressões (e2) e (e3) - e determinar $\mathrm{C}_{\mathrm{D}}$, conhecendo-se S. S foi estimado de acordo com Kolmogorov e Duplishcheva (10), através da potência $2 / 3$ do volume corporal, por sua vez inferido do peso e da altura do nadador.

Dos resultados destacam-se, portanto, os valores de $\mathrm{D}$ e de $\mathrm{P}$, determinados com um erro potencial máximo da ordem dos 6 a $8 \%$ (10). $C_{D}$ constitui, portanto, uma entidade que devem ser entendida como menos robusta.

\subsection{Tratamento estatístico}

Os resultados foram tratados através das medidas descritivas habituais (média e desvio padrão). O significado estatístico das diferenças de médias foi estudado de duas formas distintas, mantendo-se sempre $\alpha=0.05$ : através da ANOVA a um factor (Scheffe F-test à posteriori) para as diferenças entre grupos de $n$ mais expressivo $(\geq 9)$ e através do teste não paramétrico de Kruskal-Wallis, para comparar os primeiros com os grupos de $\mathrm{n}$ reduzido, bem como estes entre si.

As relações entre variáveis foram pesquisadas através da regressão linear e dos coeficientes de determinação e de correlação de Pearson.

\section{RESULTADOS E DISCUSSÃO}

Os principais resultados do estudo são apresentados nas Figuras 4 a 8.

$\mathrm{Na}$ Figura 4 percebe-se que os valores de $\mathrm{D}$ à velocidade máxima de nado foram inferiores nos nadadores pré-juniores do primeiro ano relativamente aos do segundo ano e aos nadadores masculinos de top internacional. Os valores das médias cresceram progressivamente entre os diferentes escalões etários e níveis desportivos em ambos os sexos, mas algumas diferenças não apresentaram significado estatístico, nomeadamente entre os grupos do sexo feminino.

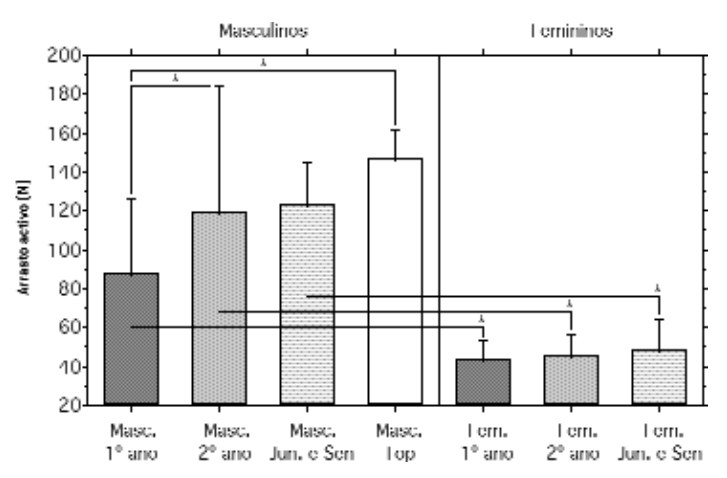

Figura 4. Variação, entre os diferentes grupos de nadadores estudados, dos valores médios e respectivos desvios-padrão do arrasto activo. $\varepsilon$ também apresentado o significado estatístico das diferenças intrasexuais e intersexuais de médias ( $^{*}=0.05$ ].

O crescimento de $\mathrm{D}$ com a idade e o nível desportivo era antecipadamente esperado, sobretudo por duas razões fundamentais: (i) com a idade - e sobretudo entre os nadadores pré-juniores e os restantes - é ainda esperado um crescimento físico assinalável, nomeadamente próximo-distal e em volume, o que presumivelmente se reflectirá num aumento de $S$, já que não parece afectar $C_{D}$ (Figura 5) e (ii) com a idade e com o nível desportivo, observa-se um natural aumento progressivo da velocidade máxima de nado (Figura 6), o que implicará também um aumento de $\mathrm{D}$, já que este parâmetro, para um mesmo sujeito ou corpo, varia sempre com a velocidade, seja com o quadrado da velocidade para escoamentos newtonianos, seja simplesmente de forma linear para escoamentos de Stokes (4).

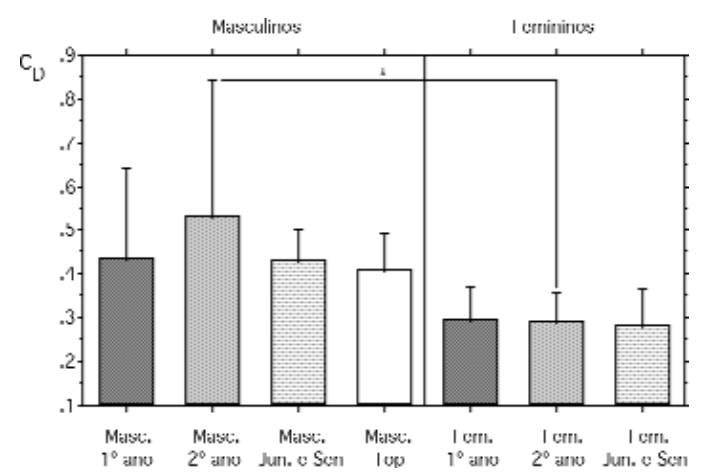

Figura 5. Variação, entre os diferentes grupos de nadadores estudados, dos valores médios e respectivos desvios-padrão do coeficiente de arrasto (CD). $\mathcal{E}$ também apresentado o significado estatístico das diferenças intra-sexuais e intersexuais de médias ( $^{*}=0.05$ ). 
A possibilidade da variação da velocidade de nado influir na comparação dos resultados relativos a D é confirmada pela relação observada entre as duas variáveis em contexto amostral (Figura 7).

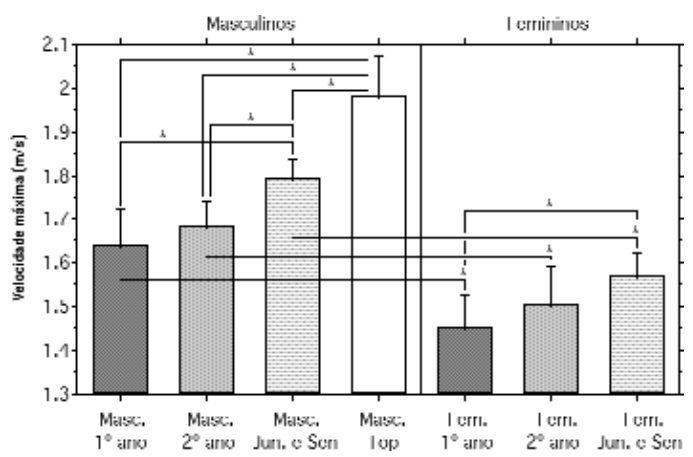

Figura 6. Variação, entre os diferentes grupos de nadadores estudados, dos valores médios e respectivos desvios-padrão da velocidade máxima de nado. $\varepsilon$ também apresentado o significado estatístico das diferenças intrasexuais e intersexuais de médias $\left(^{*}=p \leq 0.05\right.$ ).

As diferenças nas dimensões das várias amostras poderá justificar a não observação de diferenças significativas onde estas poderiam ser esperadas mediante a simples comparação da expressão puramente gráfica das médias e respectivos desvios-padrão.

Os valores absolutos observados são coerentes com os anteriormente publicados na literatura para nadadores de elite utilizando o mesmo método (10), ou utilizando o MAD-System (14), especialmente no que concerne às diferenças intersexuais observadas $(10,13)$.

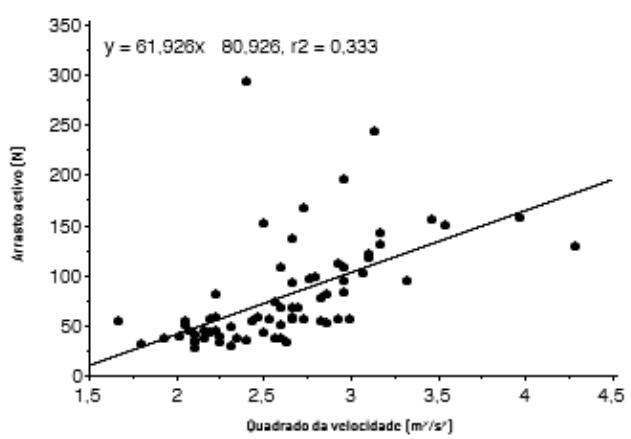

Figura 7. Regressão linear entre o arrasto activo e o quadrado da velocidade máxima de nado $(r=0.577, p \leq 0.05)$.
Valores inferiores de D nas mulheres foram imputados às menores velocidades de nado, mas também a valores inferiores das dimensões corporais (1) e, sobretudo, a uma maior flutuabilidade e menor momento hidrostático (13). Jurina (6) defendeu ainda a possibilidade da forma corporal da mulher poder ser especialmente mais hidrodinâmica do que a do homem, hipótese que poderá parecer coerente com os nossos resultados, apesar de só se ter observado uma diferença intersexual significativa (Figura 5). Todavia, Kolmogorov et al. (11) não registaram diferenças intersexuais significativas em $C_{D}$ para arrasto activo, sugerindo que este poderá ser principalmente determinado pela técnica de nado e menos pela morfologia do nadador. Nesta perspectiva, a ainda que muito ténue tendência para uma progressiva redução dos valores de $\mathrm{C}_{\mathrm{D}}$ com a idade e com o nível desportivo em cada sexo (Figura 5) é muito interessante e sugere que as pressões selectivas, em natação, podem passar pelas variáveis que determinam uma redução de $\mathrm{C}_{\mathrm{D}}$, quer a nível morfológico, quer a nível técnico.

A potência mecânica propulsiva máxima (Figura 8) tendeu a crescer significativamente, com a idade e o nível desportivo, entre os nadadores do sexo masculino.

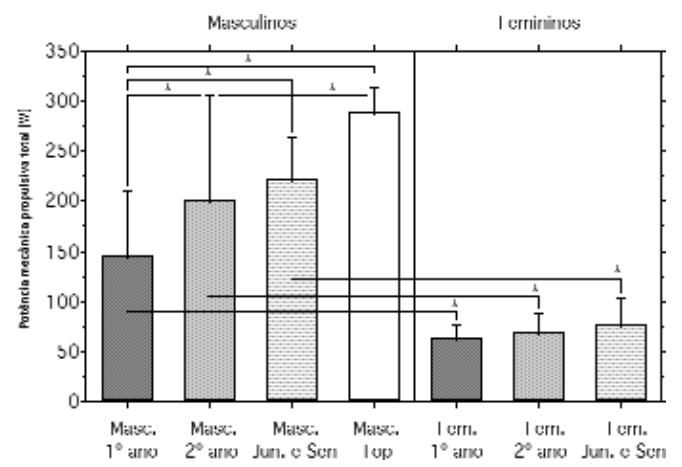

Figura 8. Variação, entre os diferentes grupos de nadadores estudados, dos valores médios e respectivos desvios-padrão da potência mecânica propulsiva máxima. $\bar{\varepsilon}$ também apresentado o significado estatístico das diferenças intra-sexuais e intersexuais de médias ( $\left.^{*}=p \leq 0.05\right]$.

O mesmo já não aconteceu entre as nadadoras, uma vez que as diferenças notadas, de tendência semelhante, não se apresentaram suficientemente robustas. As nítidas diferenças intersexuais observadas neste parâmetro, são conformes a resultados anterio- 
res e às diferenças intersexuais largamente descritas no domínio das capacidades físicas reportadas à potência mecânica, nomeadamente no domínio da força (10). Estas, entretanto, constituem o argumento maior que pode sustentar a possibilidade dos nadadores nadarem de forma mais veloz do que as nadadoras (Figura 6), apesar de constrangidos por dimensões corporais normalmente superiores $[>S$, cf. expressão (e4)] e por valores de $C_{D}$ também tendencialmente mais elevados (Figura 5).

\section{CONCLUSÕES}

As principais conclusões deste estudo foram:

(i) os valores de $\mathrm{D}$ à velocidade máxima de nado são inferiores nos nadadores pré-juniores do primeiro ano aos dos do segundo ano e dos nadadores masculinos de top internacional, evidenciando uma tendência em ambos os sexos para crescer com o nível desportivo e com o escalão etário, de forma semelhante à do crescimento da velocidade máxima;

(ii) os valores de $\mathrm{D}$ à velocidade máxima de nado são mais elevados nos nadadores do que nas nadadoras;

(iii) a velocidade máxima de nado conseguida pelos nadadores pré-juniores é superior à conseguida pelas nadadoras e é inferior à dos nadadores mais velhos e de nível desportivo mais elevado;

(iv) os valores de $C_{D}$ tendem a ser superiores nos nadadores pré-juniores relativamente às nadadoras do mesmo escalão e não variam expressivamente com o escalão etário e nível desportivo e

(v) os valores da potência mecânica propulsiva máxima $(\mathrm{P})$ crescem entre os nadadores com a idade e nível desportivo e são superiores nos nadadores relativamente aos seus pares do sexo feminino.

\author{
CORRESPONDÊNCIA \\ João Paulo Vilas-Boas \\ Laboratório de Biomecânica \\ Faculdade de Ciências do Desporto \\ e de Educação Física \\ Rua Dr. Plácido Costa, 91 \\ 4200 Porto \\ Portugal \\ jpvb@fcdef.up.pt
}




\section{REFERÊNCIAS}

1. Chatard JC, Collomp C, Maglischo E, Maglischo C (1990). Swimming skill and stroking characteristics of front crawl swimmers. Int J Sports Med 11(2): 116-161

2. Clarys J (1979). Human morphology and hydrodynamics. In: Terauds J, Bedingfield EW (eds.), Swimming III. Baltimore: University Park Press, 3-41

3. Di Prampero PE, Pendergast DR, Wilson DW, Rennie DW

(1974). Energetics of swimming in man. J Appl Physiol 37: 1-5

4. Fédiaevsky C, Voïtkounski I, Faddéev Y (1979). Mecânica dos fluidos. Porto: Lopes da Silva

5. Hollander AP, de Groot G, van Ingen Schenau GJ (1985).

Active drag in female swimmers. Abstracts of the 10th Congress of Biomechanics. ISB

6. Jurina K (1972). Estudio comparativo del nadar del pez y el del hombre. Novedades em natacíon 1:19-28. Madrid: INEF

7. Karpovich PV (1933). Water resistance in swimming. Res Quart. 4: 21-28

8. Kemper HCG, Verschuur R, Clarys JP, Jiskoot J, Rijken H (1976). Efficiency of swimming the front crawl. In: Komi PV (ed.), Biomechanics VB. Baltimore: University Park Press, 243249

9. Kemper HCG, Verschuur R, Clarys JP, Jiskoot J (1983). Total efficiency and swimming drag in swimming the front crawl. In Hollander AP, Huijing PA, de Groot G (eds.), Biomechanics and medicine in swimming. Champaign, Illinois: Human Kinetics, 199-206

10. Kolmogorov SV, Duplishcheva OA (1992). Active drag, useful mechanical power output and hydrodynamic force coefficient in different swimming strokes at maximal velocity. $J$ Biomechanics 25(3): 311-318

11. Kolmogorov SV, Rumyantseva OA, Gordon BJ, Cappaert JM (1997). Hydrodynamic characteristics of competitive swimmers of different genders and performance levels. J Appl Biomech 13: 88-97

12. Liljestrand G, Stenström N (1919). Studiën uber die physiologie des chwimmens. Scand Arch Physiol 39: 1-63

13. Rennie DW, Pendergast DR, di Prampero PE (1975).

Energetics of swimming in man. In: Clarys JP, Lewillie L (eds.) Swimming II. Baltimore: University Park Press, 97-104

14. Toussaint HM (1988). Mechanics and energetics of swimming. Tese de doutoramento. Universidade Livre de Amesterdão, Amesterdão

15. Ungerechts B, Niklas A (1994). Factors of active drag estimated by flume swimming. In: Miyashita M, Mutoh M,

Richardson R (eds.), Medicine and science in aquatic sports, Med Sport Sci 39: 137-142. Basel: Karger. 